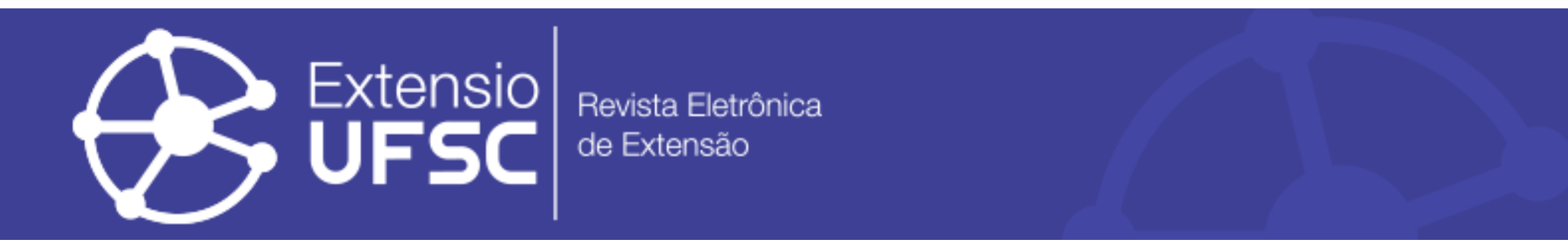

\title{
AS FASES DE ADAPTAÇÃO NO CUIDAR: INTERVENÇÕES COM CUIDADORES FAMILIARES NO DOMICÍLIO
}

\author{
Stefanie Griebeler Oliveira \\ Universidade Federal de Pelotas \\ stefaniegriebeleroliveira@gmail.com \\ Michele Rodrigues Fonseca \\ Universidade Federal de Pelotas \\ michelerf@bol.com.br \\ Maiara Simões Formentin \\ Universidade Federal de Pelotas \\ maiaraformentinn@gmail.com
}

Andriara Canez Cardoso Universidade Federal de Pelotas andriaraccardoso@gmail.com

Mateus Menezes Ribeiro Universidade Federal de Pelotas mts2529@gmail.com

Adrize Rutz Porto Universidade Federal de Pelotas adrizeporto@gmail.com

Zayanna Christine Lopes Lindôso Universidade Federal de Pelotas zayannaufpel@gmail.com

\section{Resumo}

A partir do Projeto de Extensão "Um olhar sobre o cuidador familiar: quem cuida merece ser cuidado", realizado com 52 cuidadores de pacientes crônicos dos Serviços de Atenção Domiciliar no sul do Rio Grande do Sul, objetivou-se relatar a experiência da identificação das fases de adaptação do processo de cuidar e intervenções realizadas aos cuidadores no contexto domiciliar. Por meio de quatro visitas domiciliares por acadêmicos de Enfermagem e da Terapia Ocupacional, no período de junho de 2015 a dezembro de 2016, produziu-se registros, nos quais a fase de resolução foi a mais frequente e a escuta terapêutica foi a intervenção mais realizada. Assim, a identificação das fases de adaptação de Ferré-Grau et al. (2011) possibilitou conhecer as necessidades do cuidador e o planejamento de ações de promoção de saúde e prevenção de agravos.

Palavras-chave: Adaptação Psicológica. Cuidadores. Serviços de Assistência Domiciliar.

\section{THE ADAPTATION PHASES OF CARE: INTERVENTIONS WITH FAMILY CAREGIVERS AT HOME}

Abstract

From the Extension Project A look at the family caregiver: who cares deserves to be taken care of, carried out with 52 caregivers of chronic patients from the Home Care Services in the south of Rio Grande do Sul, it was aimed to report the experience of identifying the phases of adaptation of the caregiving process and interventions performed to caregivers in the home context. Though four home visits by Nursing and Occupational Therapy students, from June 2015 to December 2016, records were produced, in which the resolution phase was the most present and the therapeutic listening was the most frequently performed intervention. Thus, the identification of the phases of adaptation of Ferré-Grau et al. (2011) made it possible to know the needs of the caregiver and the planning of actions of health promotion and harm prevention. Keywords: Adaptation. Psychological. Caregivers. Home Care Services.

\section{LAS FASES DE ADAPTACIÓN EN CUIDAR: INTERVENCIONES CON CUIDADORES FAMILIARES EN EL DOMICILIO}

\section{Resumen}

A partir del Proyecto de Extensión Un mirar sobre el cuidador familiar: quién cuida merece ser cuidado, realizado con 52 cuidadores de pacientes crónicos de los Servicios de Atención Domiciliar en el sur de Rio Grande do Sul, se objetivó relatar la experiencia de la identificación de las fases de adaptación del proceso de cuidar e intervenciones realizadas a los cuidadores en el contexto domiciliar. Por medio de cuatro visitas domiciliarias por académicos de Enfermería y de la Terapia Ocupacional, en el período de junio de 2015 a diciembre de 2016, se produjeron registros, en los cuales la fase de resolución fue la más frecuente y la escucha terapéutica fue la intervención más realizada. Así, la identificación de las fases de adaptación de Ferré-Grau et al. (2011) posibilitó conocer las necesidades del cuidador y la planificación de acciones de promoción de salud y prevención de agravios.

Palabras-clave: Adaptación Psicológica. Cuidadores. Servicios de Atención de Salud a Domicilio. 
As fases de adaptação no cuidar: intervenções com cuidadores familiares no domicílio

\section{INTRODUÇÃO}

O desenvolvimento da tecnologia médica, dos diagnósticos e tratamentos, acompanhados do aumento da expectativa de vida da população, não têm trazido garantias de cuidado com qualidade (GUIMARÃES; LIPP, 2011). Por esse motivo, há necessidade dos serviços de Atenção Domiciliar que visem a prestação e integralização do cuidado ao paciente no domicílio. Esses serviços constituem-se em uma nova modalidade de atenção à saúde, substitutiva ou complementar às já existentes, e representam um conjunto de ações de promoção da saúde, prevenção e tratamento de doenças e reabilitação da saúde desenvolvidas no domicílio, com garantia de continuidade de cuidados e integrado às redes de atenção à saúde (BRASIL; 2016).

Nessa direção, as visitas domiciliares são importantes instrumentos de atenção à saúde no âmbito domiciliar, pois proporcionam o conhecimento da realidade do paciente, familiar e cuidador (DUARTE; DIOGO, 2000). O cuidador da pessoa em adoecimento, geralmente é o familiar mais próximo, sendo fundamental na prestação dos cuidados no processo saúde e doença do paciente, e, por este motivo, tem a sua rotina transformada, devido às diversas responsabilizações relacionadas a estes cuidados (RIBEIRO et al., 2017).

É preciso atentar para as questões que envolvem a família que executa os cuidados e assumem o papel institucional dos serviços de saúde de promover a recuperação, o conforto, o alívio do sofrimento, com apoio e controle da equipe multiprofissional de atenção domiciliar (OLIVEIRA; KRUSE, 2017), pois tais cuidadores tornam-se suscetíveis, por exemplo, a sobrecarga emocional e a privações de necessidades humanas básicas como sono e boa alimentação, vivendo em isolamento social por ficar em torno do paciente e longe de suas atividades ocupacionais (OLIVEIRA et al., 2015a; OLIVEIRA et al., 2016).

Portanto, é preciso pensar formas de abordar o cuidador familiar contemplando suas necessidades durante o cuidar. Uma das estratégias utilizadas pelo Projeto de Extensão "Um olhar sobre o cuidador familiar: quem cuida merece ser cuidado" consiste na identificação da fase de adaptação do processo de cuidar em que o cuidador familiar se encontra. As fases de adaptação são: Negação; Busca de informação; Reorganização e Resolução (FERRÉ-GRAU et al., 2011).

A primeira fase consiste em uma reação psicológica de autoproteção, a qual permite ao cuidador controlar seus medos e ansiedades, pois faz com que ele se afaste da ameaça e incerteza da doença que afeta seu familiar. É um tempo necessário para que o cuidador perceba as dificuldades que o paciente apresenta. Se prolongada, se converte em fuga, e impede que o cuidador avance no processo de cuidado (FERRÉ-GRAU et al., 2011). 
As fases de adaptação no cuidar: intervenções com cuidadores familiares no domicílio

$\mathrm{Na}$ segunda fase, ocorre aceitação da realidade do doente pelo cuidador, mas podem aparecer sentimentos de angústia, raiva, culpa e frustração e vitimização devido à situação que creem não merecer. Já na terceira fase, a chamada reorganização, a vida dos cuidadores se reorganiza com o passar do tempo, mesmo que persistam alguns sentimentos como raiva, frustração, solidão em face à condição do paciente. O cuidador sente que possui mais controle das circunstâncias e aceita as modificações que a situação comporta, pois adequa sua vida conforme as necessidades do paciente (FERRÉ-GRAU et al., 2011).

Por último, na resolução, os cuidadores apresentam-se mais tranquilos, apesar das dificuldades permanecerem, são capazes de atender com êxito as demandas dos cuidados presentes e futuros (FERRÉ-GRAU et al., 2011). O conhecimento das fases de adaptação do cuidador ao cuidar possibilita que profissionais de saúde planejem e efetuem intervenções sobre as dificuldades peculiares a cada momento vivenciado no processo de cuidado ao paciente.

Assim, torna-se relevante compartilhar experiências de abordagem e intervenções com cuidadores familiares, uma vez que há poucas publicações que indicam proposições que amenizem a sobrecarga desse ser, que é fundamental para o acompanhamento do paciente no domicílio (VELLEDA; SARTOR; OLIVEIRA, 2014). Deste modo, o objetivo deste trabalho consiste em relatar a experiência da identificação das fases de adaptação do processo de cuidar e intervenções realizadas aos cuidadores no contexto domiciliar na cidade de Peloras/RS.

\section{MATERIAIS E MÉTODOS}

Trata-se de um relato de experiência, a partir do Projeto de Extensão "Um olhar sobre o cuidador familiar: quem cuida merece ser cuidado", que teve sua elaboração em meados de 2014, e início de execução em junho de 2015. O projeto de extensão é composto e executado por acadêmicos da Faculdade de Enfermagem e do Curso de Terapia Ocupacional da Universidade Federal de Pelotas (UFPel). Tal projeto foi aprovado pelo Hospital Escola (HE) da UFPEL para sua execução e inserção acadêmica nos Serviços de Atenção Domiciliar (SAD) dessa instituição, tais como: Programa de Internação Domiciliar Interdisciplinar (PIDI) Oncológico e o Programa Melhor em Casa, para acessar informações dos cuidadores potenciais para participar das ações extensionistas.

O PIDI, criado em 2005, possui atualmente duas equipes compostas por médicos clínicos, enfermeiros e técnicos de enfermagem, que atendem diariamente a pacientes em suas residências. O apoio complementar é fornecido por meio da atuação de assistentes sociais, nutricionistas, fisioterapeutas, psicólogos, farmacêuticos e auxiliar administrativo. Cada equipe 
As fases de adaptação no cuidar: intervenções com cuidadores familiares no domicílio

visita duas vezes ao dia dez pacientes. Já o programa Melhor em Casa iniciou em 2012 e conta três Equipes Multiprofissionais de Atenção Domiciliar (EMAD) - formadas por médicos, enfermeiros, técnicos em enfermagem e assistentes sociais - e uma Equipe Multiprofissional de Apoio (EMAP) - formada por fisioterapeuta, nutricionista e psicólogo que realizam os atendimentos uma a três vezes por semana e em média 30 pacientes, totalizando 150 pacientes nesse programa.

Foram convidados para participar do projeto cuidadores familiares de pacientes vinculados aos SAD do HE da UFPEL, na cidade Pelotas/RS. Para serem potenciais participantes, também deveriam ser maiores de 18 anos e cuidadores de paciente adulto que apresentasse condição crônica e/ou estivesse em situação de terminalidade. Destaca-se que, os cuidadores que foram convidados para participar do projeto receberam esclarecimentos sobre ele e informados que poderiam recusar a participação ou retirar-se sem prejuizos, quando assim o decidissem.

Os dados que compõem o presente relato de experiência foram coletados através dos registros realizados nas fichas cadastrais de cada cuidador acompanhado pelo projeto acerca das experiências e reflexões relatadas por eles nos encontros. Tais encontros foram realizados no período de junho de 2015 a novembro de 2016, nos quais foram acompanhados um total de 52 cuidadores familiares, todos residentes na cidade de Pelotas.

As visitas domiciliares foram realizadas por trios ou quartetos formados pelos acadêmicos, em quatro encontros sistematizados com escuta terapêutica, proporcionados semanalmente, com duração média de 40 minutos. Cada encontro possui um foco, sendo o primeiro desenvolvido a partir de uma ficha de cadastro com dados sociodemográficos e levantamento da história familiar, dificuldades e facilidades enfrentadas durante o processo de cuidado e como foi designado o papel de cuidador.

No segundo encontro, utilizou-se um vídeo com imagens em desenho relacionadas ao cotidiano do cuidado (Figura 1) produzidas por uma acadêmica de Enfermagem, durante o primeiro semestre de 2015. Tal vídeo foi elaborado com intenção de produzir reflexões relacionadas ao cuidado. 


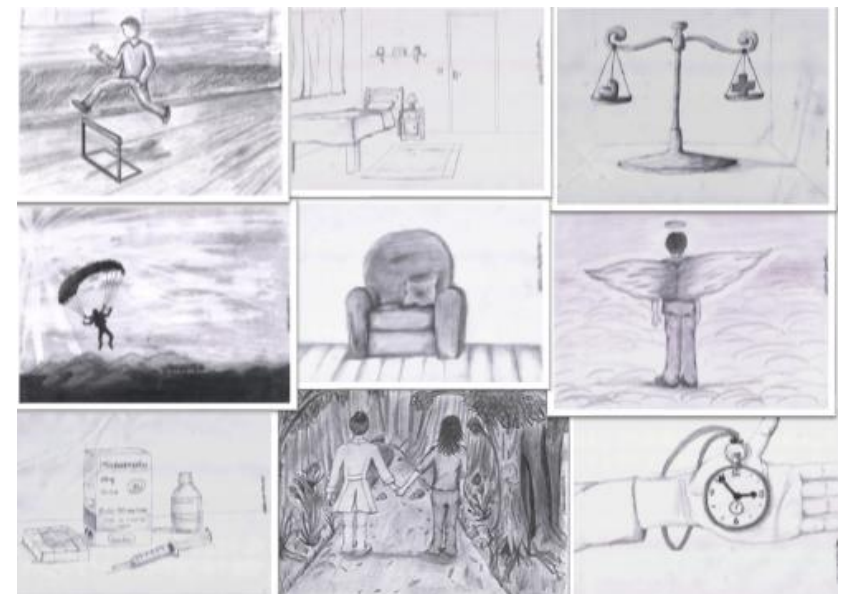

Figura 1. Desenhos produzidos para o vídeo do Projeto de Extensão, pela acadêmica Caroline de Melo Oreste

No terceiro encontro, questionou-se acerca das imagens do vídeo, se instigaram mais reflexões em relação às suas práticas no intervalo dos encontros. Retomou-se temas como desafios, enfrentamentos, fragilidades e potencialidades da experiência do cuidar, de modo a facilitar a identificação da “fase de adaptação do cuidado"(FERRÉ-GRAU et al., 2011) em que o cuidador se encontra. Tal identificação foi realizada a partir da relação do conteúdo que compõe cada fase (FERRÉ-GRAU et al., 2011), conforme descreve-se na introdução deste artigo, com o que o cuidador relatou. A partir dessa identificação por aproximação que as intervenções (Figura 2) foram selecionadas e executadas, baseadas nas necessidades apresentadas conforme a definição da fase. Algumas intervenções foram inspiradas em Ferré-Grau et al. (2011) e outras foram elaboradas pelo grupo do projeto de extensão, nas reuniões em que os casos acompanhados eram discutidos, conforme os campos comuns de saber entre enfermagem e terapia ocupacional, e os campos específicos de cada uma destas formações e/ou profissões.

\begin{tabular}{|c|c|}
\hline \multicolumn{2}{|c|}{ INTERVENÇÕES SUGERIDAS E REALIZADAS } \\
\hline $\begin{array}{c}\text { 1. Realizar escutar de forma terapêutica***; } \\
\text { 2.Estreitar o vínculo entre cuidador e Unidade } \\
\text { Básica de Saúde (UBS)**; } \\
\text { 3.Encaminhar para acompanhamento com } \\
\text { psicólogo***; } \\
\text { 4.Verificar pressão arterial***; } \\
\text { 5.Orientar e estimular a autonomia e cuidado de si, } \\
\text { destacando que para cuidar bem é necessário se } \\
\text { cuidar também***; } \\
\text { 6.Orientar para que reflita sobre as situações que } \\
\text { lhe causem estresse e buscar soluções razoáveis**; } \\
\text { 7.Encaminhar para exames***; } \\
\text { 8. Orientar a realização de exercícios físicos para } \\
\text { relaxamento muscular***; } \\
\text { 9. Orientar sobre a importância e auxiliar nas } \\
\text { mudanças de decúbito de forma correta e fácil } \\
\text { 10. Orientar sobre serviços de massagem } \\
\text { terapêutica***; } \\
\text { 11. Incentivar a leitura***; }\end{array}$ & $\begin{array}{l}\text { 21.Reforçar sentimentos positivos e conversar com } \\
\text { outros sobre os negativos***; } \\
\text { 22.Atentar-se para sintomas que indiquem } \\
\text { sobrecarga e estresse***; } \\
\text { 23. Conhecer os demais integrantes da família***; } \\
\text { 24. Facilitar e informar sobre questões sociais e } \\
\text { sobre o processo de separação (em caso de } \\
\text { terminalidade) }{ }^{* *} \text {; } \\
\text { 25.Ajudar nas decisões terapêuticas***; } \\
\text { 26.Reconhecer o esforço do cuidador nas ações***; } \\
\text { 27.Convidar para participar de atividades lúdicas em } \\
\text { ambiente favorável (Unidade Cuidativa) } * * * ; \\
\text { 28. Ofertar abordagem individual em ambiente } \\
\text { protegido (Unidade Cuidativa) }{ }^{* * * ;} \\
\text { 29. Realizar exame físico do pés em cuidadores com } \\
\text { diabetes*; } \\
\text { 30. Realizar exame físico para avaliação de } \\
\text { enfermagem, conforme queixa referida pelo } \\
\text { cuidador*; }\end{array}$ \\
\hline
\end{tabular}




\begin{tabular}{|c|c|}
\hline $\begin{array}{l}\text { 12. Identificar o exercício da escrita de si e } \\
\text { estimular a prática***; } \\
\text { 13. Explicar sobre a importância do uso correto de } \\
\text { medicamentos e da organização das medicações***; } \\
\text { 14. Orientar sobre religiosidade e espiritualidade***; } \\
\text { 15. Adequar um plano de cuidados com orientações } \\
\text { sobre organização da vida e de tarefas importantes, } \\
\text { contando com o apoio do restante da família, } \\
\text { amigos ou profissionais de saúde***; } \\
\text { 16. Explorar os apoios formais e informais do } \\
\text { cuidador identificados no ecomapa, como } \\
\text { instituições de lazer, espiritualidade, saúde, } \\
\text { relaxamento etc. } * * * ; \\
\text { 17. Identificar situações de risco, atentando-se aos } \\
\text { fatores que podem deixar o cuidador vulnerável***; } \\
\text { 18. Destacar a importância da boa comunicação } \\
\text { com o paciente, tendo paciência, sempre } \\
\text { respeitando e estimulando sua individualidade***; } \\
\text { 19. Estimular o fortalecimento de vínculo entre o } \\
\text { cuidador e o indivíduo cuidado***; } \\
\text { 20. Orientar quanto à manutenção da } \\
\text { independência do indivíduo cuidado: quando for } \\
\text { possível, estimular que o indivíduo cuidado realize } \\
\text { o máximo de atividades sozinho (sob supervisão e } \\
\text { orientações do cuidador), aumentando sua } \\
\text { autonomia e evitando a sobrecarga do cuidador***; }\end{array}$ & $\begin{array}{l}\text { 31. Orientar sobre realização de curativos e avaliar } \\
\text { lesões*; } \\
\text { 32. Planejar e preparar para a despedida**; } \\
\text { 33. Resgatar atividades significativas paralelas a } \\
\text { reorganização da rotina**; } \\
\text { 34. Proporcionar momentos de comunicação, } \\
\text { criação e expressão**; } \\
\text { 35. Ressignificar história de vida através de } \\
\text { estímulos sensoriais**; } \\
\text { 36. Auxiliar no controle físico (posicionamento, } \\
\text { órteses) e de sintomas (controle de dor, } \\
\text { conservação de energia...)**; } \\
\text { 37. Adaptar tarefas e utensílios para o desempenho } \\
\text { seguro das atividades da vida diária (AVD)**; } \\
\text { 38. Esclarecer, quando necessário, sobre quadros } \\
\text { clínicos do ponto de vista terapêutico } \\
\text { ocupacional**; } \\
\text { 39. Capacitar quanto a manobras e equipamentos } \\
\text { adaptativos (tecnologia assistiva) para a realização } \\
\text { das atividades de vida diária (higiene, alimentação, } \\
\text { vestuário, locomoção e comunicação), respeitando } \\
\text { os desejos do indivíduo cuidado**. }\end{array}$ \\
\hline
\end{tabular}
intervenções do campo da Terapia Ocupacional; *** intervenções comuns aos acadêmicos de enfermagem e da Terapia ocupacional).

Fonte: dados projeto de extensão (OLIVEIRA et al., 2015b).

No quarto e último encontro, foram desenvolvidas as intervenções e avaliou-se a partir de questionamentos ao cuidador acerca das atividades realizadas e sobre como se sentiram durante os encontros com os acadêmicos.

\section{RESULTADOS E ANÁLISES}

Os registros produzidos nas visitas domiciliares, especialmente no terceiro encontro, continham informações sobre a experiência de cuidar, bem como os desafios e os anseios que ainda permaneciam, sendo possível identificar a fase de adaptação do processo de cuidar do cuidador familiar, no vínculo construído entre ele e o acadêmico (FERRÉ-GRAU et al., 2011). Estas autoras descrevem as quatro fases, mencionadas na introdução deste artigo, indicando que podemos identificá-las em cuidadores que acompanhamos, por aproximação das informações, ou seja, a partir dos dados que coletamos dos cuidadores, apontou-se as características que estão descritas em cada uma das fases. A identificação de tal fase possibilita que acadêmicos e/ou profissionais planejem intervenções de cuidado mais acertadas ao momento e às necessidades dos cuidadores. 
As fases de adaptação no cuidar: intervenções com cuidadores familiares no domicílio

Entre os 52 cuidadores familiares acompanhados no período de junho de 2015 a novembro de 2016, constatou-se que sete estavam na primeira fase de adaptação - negação; três cuidadores se encontravam na segunda fase - busca de informações; 13 cuidadores na terceira fase - reorganização; e, por fim, 21 na última fase - resolução. Oito cuidadores não foram acompanhados nos quatro encontros previstos pelo projeto devido à desistência destes e/ou pelo falecimento do paciente, portanto, esses dados não foram considerados pela insuficiência de informações para a identificação das fases de adaptação.

Os cuidadores, identificados na fase de negação, apresentaram inconformações, incertezas e incompreensões com a situação, uma vez que não esperam se tornar um cuidador familiar. Para Ferré-Grau et al. (2011), lidar com mudanças repentinas não é uma tarefa simples e isso exige tempo ao cuidador para que ele se adapte aos cuidados necessários ao paciente. No entanto, compreendemos, a partir do acompanhamento realizado com os cuidadores, que a fase de negação tem sua produtividade, no que se refere a manter o cuidador, de certa forma, fortalecido para assumir este papel, pois embora apresente incertezas da situação ou as negue, permite que ele não fique fragilizado emocionalmente a ponto de impossibilitá-lo no desenvolvimento das ações de cuidar. Assim, foram desenvolvidas as intervenções descritas na Figura 2, representadas pela numeração: 1, 3, 6, 8, 14, 16, 17, 19, 21, 22, 36, 37, 39 .

Em um estudo de Castro et al (2015), os cuidadores demonstraram que embora tivessem aceitado cuidar de seu familiar, e que no desenvolvimento das ações sempre tenham tentado dar o melhor de si para ofertar conforto e bem-estar ao paciente, muitas vezes não são capazes de esquecer os conflitos relacionais vivenciados em outras etapas da vida de ambos. Esta é uma questão interessante que também é apontada em outro estudo por Mendes e Santos (2016), no qual foi identificado entre os cuidadores sentimentos conflituosos de negação, comoção, culpa, obrigação e reclusão. A partir disso, a enfermagem tem potencial para atuar identificando as causas que produzem tais sentimentos, auxiliando os cuidadores a reconhecê-los e ajudando no planejamento de alterações na rotina que possam contribuir para a diminuição da tensão emocional.

O tempo da experiência de cuidado também influencia, de certo modo, a consciência do cuidador em relação à terminalidade do paciente. Por exemplo, nos primeiros meses de cuidado ao paciente no domicílio, o cuidador busca saber pouco ou nada sobre o prognóstico ruim, repetindo o que foi dito pelo médico e, por fim, assumindo que não deseja saber informações relacionadas à doença ou sua progressão (OLIVEIRA et al., 2012).

Os cuidadores que se encontravam na segunda fase de adaptação buscavam informações acerca das incertezas e dúvidas quanto à doença e as ações de cuidado. Embora na primeira fase 
As fases de adaptação no cuidar: intervenções com cuidadores familiares no domicílio

haja incertezas, o cuidador não busca informações acerca do contexto vivenciado. Esta é a diferença, conforme referem Ferré-Grau et al. (2011), entre uma fase e a outra. Todavia, essa necessidade de acessar as informações na segunda fase pode gerar no cuidador pessimismo e tristeza, e é nesse sentido que as interveções devem ser organizadas e planejadas, para dar suporte a ele, possibilitando que se sinta mais seguro para prestar o cuidado ao paciente, conseguindo, assim, assumir-se como um cuidador familiar. Nesse sentido, foram desenvolvidas as seguintes intervenções: 1, 3, 5, 6, 8, 9, 11, 12, 13, 14, 15, 16, 17, 18, 19, 20, 21, 22, 24, 26, 31, 36, 38, 39.

Esta segunda fase costuma ocorrer no primeiro ano da experiência de cuidado, e conforme um estudo (OLIVEIRA et al., 2012), os significados produzidos pelos cuidadores em torno da terminalidade do paciente oscilam entre esperança de cura da doença e a aceitação do prognóstico ruim. Ora, buscam-se todas as alternativas possíveis que ofertem ou prometam cura, e ora, racionaliza-se que a proximidade da morte do paciente é inevitável, mas que diante de alguma intercorrência, como na fase agônica, por exemplo, a cuidadora o levaria a um serviço de saúde.

Os cuidadores que foram identificados na terceira fase, denominada reorganização, já estavam há mais de um ano realizando esse papel. Percebemos que eles se encontravam mais seguros sobre o cuidado ao paciente, mesmo alguns apresentando alguns aspectos de sobrecarga. Essas questões diferiram das fases anteriores, pois não impediam o cuidador de traçar alguns objetivos pessoais e retomar algumas atividades realizadas na sua rotina anterior, o que os deixou mais contentes. Em suma, Ferré-Grau et al (2011) apontam que, nesta fase, o cuidador consegue adaptar a rotina de cuidado a alguns hábitos tidos antes de assumir tal papel.

Destacamos que o fator tempo parece ter importante relação com essa fase de adaptação, ou seja, quanto maior o período da experiência de cuidado ao paciente, mais o cuidador sente-se seguro e consegue se organizar para retomar algumas de suas atividades que lhe proporcionavam prazer, atenuando a sobrecarga. Nesse sentido, se trabalharmos intervenções junto ao cuidador que se encontra nas fases iniciais, podemos propiciar melhor e mais rápida elaboração da primeira e segunda fase para que ele chegue em um período menor de um ano à terceira fase, melhorando, assim, sua qualidade de vida.

A experiência de cuidar por mais de um ano também facilita a aceitação da terminalidade do paciente, permitindo que o cuidador participe das decisões terapêuticas de forma mais crítica em relação aos procedimentos invasivos (OLIVEIRA et al., 2012). Nesse sentido, para esta fase, as intervenções elencadas foram: 1, 2, 5, 6, 7, 8, 10, 11, 18, 20, 23, 25, 27, 28, 30, 33, 34.

Entre os cuidadores que se encontravam na última fase, a de resolução, observamos que além do cuidador manter suas relações com familiares e com amigos, ele conserva a sua 
As fases de adaptação no cuidar: intervenções com cuidadores familiares no domicílio

espiritualidade e estabelece prioridades para si e para o paciente, preservando e adequando melhorias ao cuidado do paciente e de si próprio, indo ao encontro do que Ferré-Grau et al (2011) definiram. Nessa fase, o cuidador conversa sobre o prognóstico do paciente, demonstrando, em seus relatos, algumas formas de organizaçao para o momento da despedida. Entre as intervenções desenvolvidas, enumeradas na Figura 2, estão: 1, 2, 5, 6, 8, 23, 24, 25, 26, $27,28,30,32,33,34,35$.

A escuta terapêutica, intervenção utilizada em todas as visitas dos acadêmicos, consiste em um método de incentivar uma melhor comunicação e compreensão das preocupações pessoais, com a necessidade por parte do ouvinte de identificar os aspectos verbais e não verbais da comunicação. A escuta é um instrumento importante para a obtenção de informações, pelo uso de perguntas abertas e esclarecimentos, e permite que o profissional ajude o cuidador a enfrentar seus problemas e ajustar o que não pode ser mudado (MESQUITA; CARVALHO, 2014).

Os cuidadores, especialmente aqueles que são os únicos responsáveis pelo cuidar, sofrem de sobrecarga emocional e psicológica. Muitas das vezes, só precisam de alguém para conversar, os ouvir, um suporte psicológico. Por isso, o vínculo que também conseguimos realizar de alguns cuidadores com o psicólogo foi essencial. Ter com quem dividir e compartilhar os cuidados, conforme estudos de Anjos et al (2015) e Oliveira et al.(2016), representa uma diminuição de estresse, apoio emocional, de trocas, de solidariedade, de um espelho que mostra um sofrimento compartilhado.

Em outros estudos sobre familiares cuidadores de pessoas com demência a residir no domićlio, as intervenções também foram nesse âmbito do apoio emocional e psicológico (MENDES; SANTOS, 2016; SOUSA et al. 2016; 2017). Outras intervenções realizadas com menor frequência foram a verificação da pressão arterial do cuidador, as orientações e esclarecimentos sobre o uso correto de medicações e a forma ergonomicamente adequada de troca de decúbito a cada duas horas dos pacientes acamados.

\section{CONSIDERAÇÕES FINAIS}

A identificação das fases de adaptação do cuidador possibilita a realização de cuidados integrais que fomentem a melhora no estado emocional, na qualidade de vida e alívio da sobrecarga, devido ao acúmulo de tarefas relacionadas ao cuidado. Nesse processo, os cuidadores podem acabar perdendo contato com as relações sociais que os cercam, como: trabalho, amigos, vizinhança e organizações religiosas, entre outras, e as ações extensionistas são capazes de estimular a aproximação dessa rede social de apoio, buscando que o cuidador retome atividades 
As fases de adaptação no cuidar: intervenções com cuidadores familiares no domicílio

de lazer e pense mais no cuidado de si. Nesse contato proporcionado pelo projeto de extensão, entre acadêmicos e cuidadores familiares, ocorreram significativas trocas de saber, em que ambas as partes têm a oportunidade de esclarecer dúvidas e solicitar informações, assim como vivenciar esse momento como uma conversa terapêutica.

\section{REFERÊNCIAS}

ANJOS, K.F. et al. Associação entre apoio social e qualidade de vida de cuidadores familiares de idosos dependentes. Ciência \& Saúde Coletiva, v. 20, n. 5, p. 1321-1330, 2015.

BRASIL. Ministério da Saúde. Portaria $n^{\circ}$ 825, de 25 de abril de 2016. Redefine a atenção domiciliar no âmbito do Sistema Único de Saúde (SUS) e atualiza as equipes habilitadas. Diário Oficial [da] União, Brasília, DF, 25 abr. 2016.

CASTRO, L. et al. Cuidador Familiar: Relação Familiar e a Satisfação no Cuidar. Investigação Qualitativa em Saúde, v.1, p. 492-496, 2015.

DUARTE, Y. A. O.; DIOGO, M. J. E. Atendimento domiciliar: um enfoque gerontológico. São Paulo: Atheneu, 2000.

FERRÉ-GRAU, C. et al. Guía de cuidados de Enfermería: Cuidar al cuidador em Atención Primaria. Imprime: Publidisa, 2011.

GUIMARÃES, C. A.; LIPP, M. E. Um olhar sobre o cuidador de pacientes oncológicos recebendo cuidados paliativos. Psicologia: Teoria e prática, v. 11, n.2, p. 50-62, 2011.

MENDES, C.F.M.; SANTOS, A.L.S. dos. O cuidado na doença de Alzheimer: as representações sociais dos cuidadores familiares. Saude e Sociedade, v. 25, n. 1, p. 121-132, 2016.

MESQUITA, A, C.; CARVALHO, E, C, de. A escuta terapêutica como estratégia de intervenção em saúde: uma revisão integrativa. Revista da Escola de Enfermagem de São Paulo, v. 48, n. 6, p. 1127-1136, 2014.

RIBEIRO, B.F. et al. Práticas de si de cuidadores familiares na atenção domiciliar. Revista Cuidarte, v. 8, n. 3, p. 1809-1825, 2017.

OLIVEIRA, S.G. et al. Internação domiciliar do paciente terminal: o olhar do cuidador familiar. Revista Gaúcha de Enfermagem, v.33, n.3, p.104-110, 2012.

OLIVEIRA S.G. et al. Enunciados sobre la atención domiciliaria en el panorama mundial: revisión narrativa. Enfermería global. v.14, n.3, p.375-389, 2015 a.

OLIVEIRA, S.G. et al. Um olhar sobre o cuidador familiar: quem cuida merece ser cuidado. Pelotas: Universidade Federal de Pelotas; Pró-Reitoria de Extensão e Cultura; Faculdade de Enfermagem, 2015b.

OLIVEIRA, S.G. et al. Representações sociais do cuidado de doentes terminais no domicílio: o olhar do cuidador familiar. Aquichan, v.16, n.3, p.359-369, 2016. 
As fases de adaptação no cuidar: intervenções com cuidadores familiares no domicílio

OLIVEIRA, S.G.; KRUSE, M.H.L. Melhor em Casa: dispositivo de segurança. Texto e Contexto Enfermagem. v.26, n.1, p.1-9, 2017.

SOUSA, L. et al . Características de um programa de capacitação para familiares cuidadores de pessoas com demência a residir no domicílio. Revista Portuguesa de Enfermagem de Saúde Mental, n. spe3, p. 33-38, 2016.

SOUSA, Lia et al . Necessidades dos cuidadores familiares de pessoas com demência a residir no domicílio: revisão integrativa. Revista Portuguesa de Enfermagem de Saúde Mental, n. spe5, p. 45-50, 2017.

VELLEDA, K. L.; SARTOR, S. F.; OLIVEIRA, S. G. Cuidados paliativos: uma reflexão sobre alternativas em prol do cuidador familiar. In: Seminário Internacional de Bioética e Saúde Pública, 2, 2014, Santa Maria. Anais: II Seminário Internacional de Bioética e Saúde Pública e II Simpósio Internacional de Ética na Pesquisa, 4, 5, 6 e 7 de junho de 2014, Santa Maria. p.227234.

Recebido em: 30/04/2017

Aceito em: 06/06/2018 